\title{
Epitaxial Growth without Slope Selection: Energetics, Coarsening, and Dynamic Scaling *
}

\author{
Bo $\mathrm{Li}^{\dagger} \quad$ Jian-Guo Liu ${ }^{\ddagger}$
}

December 27, 2004

\begin{abstract}
We study a continuum model for epitaxial growth of thin films in which the slope of mound structure of film surface increases. This model is a diffusion equation for the surface height profile $h$ which is assumed to satisfy the periodic boundary condition. The equation happens to possess a Lyapunov or "free energy" functional. This functional consists of the term $|\Delta h|^{2}$ that represents the surface diffusion and $-\log \left(1+|\nabla h|^{2}\right)$ that describes the effect of kinetic asymmetry in the adatom attachment-detachment. We first prove for large time $t$ that the interface width - the standard deviation of the height profile - is bounded above by $O\left(t^{1 / 2}\right)$, the averaged gradient is bounded above by $O\left(t^{1 / 4}\right)$, and the averaged energy is bounded below by $O(-\log t)$. We then consider a small coefficient $\varepsilon^{2}$ of $|\Delta h|^{2}$ with $\varepsilon=1 / L$ and $L$ the linear size of the underlying system, and study the energy asymptotics in the large system limit $\varepsilon \rightarrow 0$. We show that global minimizers of the "free energy" functional exist for each $\varepsilon>0$, the $L^{2}$-norm of the gradient of any global minimizer scales as $O(1 / \varepsilon)$, and the global minimum energy scales as $O(\log \varepsilon)$. The existence of global energy minimizers and a scaling argument are used to construct a sequence of equilibrium solutions with different wavelength. Finally, we apply our minimum energy estimates to derive bounds in terms of the linear system size $L$ for the saturation interface width and the corresponding saturation time.
\end{abstract}

2000 MSC: 35K55, 35Q99, 74K35, 82D25.

2003 PACS: 68.35.Ct; 68.43.Jk; 81.15.Aa.

Key words and phrases: epitaxial growth, coarsening, interface width, dynamic scaling, energy minimization, equilibrium.

*Abbreviated title: Epitaxial Growth without Slope Selection.

†Department of Mathematics, University of California at San Diego, 9500 Gilman Drive, La Jolla, CA 92093-0112, USA. E-mail: bli@math.ucsd.edu.

†Department of Mathematics and Institute for Physical Science and Technology, University of Maryland, College Park, MD 20742-4015, USA. E-mail: jliu@math.umd.edu. 


\section{Introduction}

In a typical layer-by-layer epitaxial growth that begins with a flat substrate, surface morphological instabilities often occur as the film thickness reaches a critical value. These instabilities manifest themselves as a sort of spinodal decomposition. As a result, the nucleation of islands starts and many nuclei appear on the film surface. Such nuclei evolve into mounds, and the mound structure coarsens. During the coarsening process, the number of mounds decreases. Experiments and numerical simulations suggest that the wellcharacterized lateral size of mounds, $\lambda(t)$, increases as $\lambda(t) \propto t^{n}$, where $t$ is the time variable and $n>0$ is a constant called the coarsening exponent. The interface width $w(t)$, which is the standard deviation of the height profile and measures the roughness of the surface, also increases as $w(t) \propto t^{\beta}$, where $\beta>0$ is a constant called the growth exponent. When the finite size of the underlying system becomes effective, the interface width saturates, and the saturation value $w_{s}=w_{s}(L)$ satisfies that $w_{s}(L) \propto L^{\alpha}$, where $L$ is the linear size of the underlying system and $\alpha>0$ is a constant called the roughness exponent. The corresponding saturation time $t_{s}=t_{s}(L)$ satisfies the dynamic scaling law $t_{s}(L) \propto L^{z}$, where $z$ is a constant called the dynamic exponent. In general, $z=\alpha / \beta$. See Figure 1.1. These scaling laws are often experimentally measurable, and contain much microscopic information. See $[2,3,6,8,12,16,21,23,25-28,32-37]$ and the references therein.

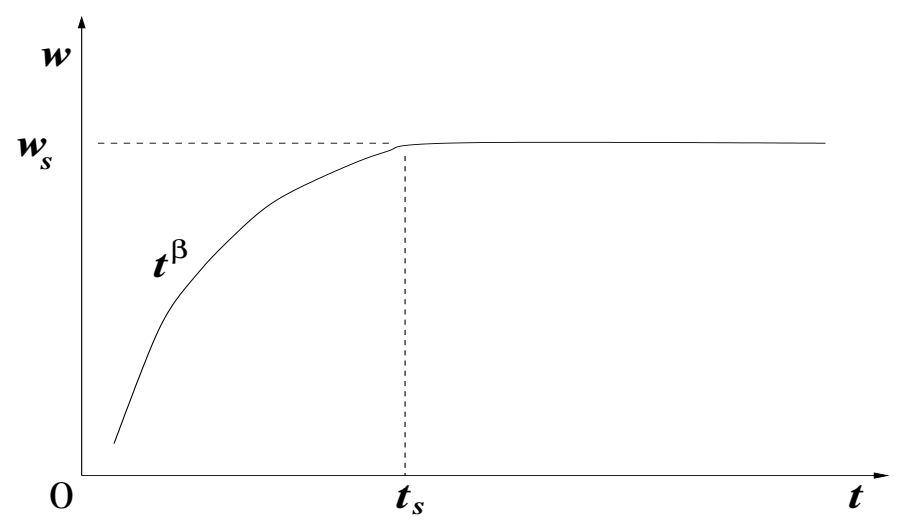

Figure 1.1. Scaling laws in epitaxial growth.

To understand these interesting phenomena, we consider in this work the diffusion equation

$$
\partial_{t} h=-\nabla \cdot\left(\frac{\nabla h}{1+|\nabla h|^{2}}\right)-\Delta^{2} h
$$

for the height profile $h$, measured in a co-moving frame, of a thin film in epitaxial growth. We assume that the height profile $h$ is spatially $\bar{\Omega}$-periodic, where $\Omega=(0, L)^{d} \subset \mathbb{R}^{d}$ is a $d$-dimensional, open cube with $d \geq 1$ an integer and $L>0$ the linear size of the cube, and $\bar{\Omega}$ is the closure of $\Omega$, i.e., $\bar{\Omega}=[0, L]^{d}$.

For epitaxial growth, $d=2$. In this case, the bi-harmonic term in Eq. (1.1) describes the surface diffusion $[10,22]$. The nonlinear, lower order term in Eq. (1.1) was first proposed 
phenomenologically in [12] to model the Ehrlich-Schwoebel effect: adatoms (adsorbed atoms) must overcome a higher energy barrier to stick to a step from an upper than a lower terrace $[5,30,31]$. See also $[17,36]$ for issues related to the model derivation. The equation describes a physical process that is far from equilibrium, and is thus not associated with the free energy of the system in the usual sense.

In general, however, Eq. (1.1) happens to possess the following Lyapunov or "free energy" functional

$$
E(h)=f_{\Omega}\left[-\frac{1}{2} \log \left(1+|\nabla h|^{2}\right)+\frac{1}{2}|\Delta h|^{2}\right] d x
$$

where

$$
f_{\Omega} u d x=\frac{1}{|\Omega|} \int_{\Omega} u d x
$$

is the averaged integral over $\Omega$ of an integrable function $u: \Omega \rightarrow \mathbb{R}$, and $|\Omega|=L^{d}$ is the $d$-dimensional volume of $\Omega$. This means that Eq. (1.1) is the gradient flow associated with the energy functional (1.2) with a suitable constant mobility. Note that we consider here, for convenience, the averaged integral in (1.2).

With the periodical boundary condition, any solution $h$ of Eq. (1.1) satisfies

$$
\frac{d}{d t} \int_{\Omega} h(x, t) d x=0
$$

i.e., the mass is conserved. For simplicity, we thus assume that the constant, spatial meanvalue of $h$ over $\Omega$ is zero. In this case, the interface width of the profile $h$ becomes

$$
w_{h}(t)=\sqrt{f_{\Omega}|h(x, t)|^{2} d x} .
$$

If $h$ is smooth and $\bar{\Omega}$-periodic, then we have by integration by parts that

$$
\begin{aligned}
\int_{\Omega}|\Delta h|^{2} d x & =\int_{\Omega}\left(\sum_{i=1}^{d} \partial_{x_{i} x_{i}} h\right)^{2} d x=\sum_{i, j=1}^{d} \int_{\Omega} \partial_{x_{i} x_{i}} h \partial_{x_{j} x_{j}} h d x \\
& =-\sum_{i, j=1}^{d} \int_{\Omega} \partial_{x_{i} x_{i} x_{j}} h \partial_{x_{j}} h d x=\sum_{i, j=1}^{d} \int_{\Omega}\left(\partial_{x_{i} x_{j}} h\right)^{2} d x
\end{aligned}
$$

Thus, the term $|\Delta h|^{2}$ in the energy functional (1.2) can be replaced by $\sum_{i, j=1}^{d}\left|\partial_{x_{i} x_{j}} h\right|^{2}$ which has all the second-order derivatives. This property follows from the periodicity along the coordinate directions, and does not imply that the surface diffusion is isotropic.

Using the identities

$$
\nabla \cdot\left(\frac{\nabla h}{1+|\nabla h|^{2}}\right)=\frac{1-|\nabla h|^{2}}{\left(1+|\nabla h|^{2}\right)^{2}} \Delta h+\frac{2|\nabla h|^{3}}{\left(1+|\nabla h|^{2}\right)^{2}} \kappa
$$


and

$$
\Delta h=\partial_{\|}^{2} h+|\nabla h| \kappa,
$$

where $\kappa=\nabla \cdot\left(\frac{\nabla h}{|\nabla h|}\right)$ is the mean curvature of level curves $h=$ constants and $\partial_{\|}=\frac{\nabla h}{|\nabla h|}$. $\nabla$ denotes the derivative tangential to the surface, we can rewrite our underlying growth equation (1.1) as

$$
h_{t}=\frac{|\nabla h|^{2}-1}{\left(1+|\nabla h|^{2}\right)^{2}} \partial_{\|}^{2} h-\frac{|\nabla h|}{1+|\nabla h|^{2}} \kappa-\Delta^{2} h .
$$

Thus, the nonlinear term in the equation describes the anisotropic diffusion in epitaxial growth: the curvature term represents the diffusion in the transverse direction and the $\partial_{\|}^{2} h$ term represents the diffusion in the direction of surface gradient.

The linearization of Eq. (1.4) around a flat surface $h_{0}(x)=m \cdot x$ for a given vector $m=\left(m_{1}, m_{2}\right) \in \mathbb{R}^{2}$ leads to the linearized equation of the growth equation $(1.1)[18,29]$

$$
\partial_{t} h=\frac{|m|^{2}-1}{\left(1+|m|^{2}\right) 2} \partial_{\| m}^{2} h-\frac{1}{1+|m|^{2}} \partial_{\perp m}^{2} h-\Delta^{2} h,
$$

where $\partial_{\| m}=m \cdot \nabla /|m|$ and $\partial_{\perp m}=m^{\perp} \cdot \nabla /|m|$ with $m^{\perp}=\left(-m_{2}, m_{1}\right)$ denote the derivatives parallel and perpendicular to the tilt vector $m$, respectively. Clearly, a singular surface $(m=0)$ is linearly unstable. The change of the sign in the coefficient of $\partial_{\| m}^{2} h$ at $|m|=1$ indicates the transition to step-flow growth. Since the coefficient of $\partial_{\perp m}^{2} h$ is always negative, the step-flow growth is linearly unstable with respect to transverse fluctuations $[4,18]$.

Heuristic scaling analysis with an assumption on the existence of scaling laws and largescale numerical simulations of this model suggest that the interface width $w(t)$ and the lateral size of mounds $\lambda(t)$ grow as $O\left(t^{1 / 2}\right)$ and $O\left(t^{1 / 4}\right)$, respectively $[8,11,24,28,33]$. Thus, the characteristic slope of mounds scales as $O\left(t^{1 / 4}\right)$ and becomes unbounded. Moreover, this noslope-selection model predicts that the saturation interface width $w_{s}(L) \propto L^{2}$. Consequently, the predicted growth, roughness, and dynamic exponents are

$$
\beta=\frac{1}{2}, \quad \alpha=2, \quad z=4 .
$$

In contrast to this model without slope selection, the model with slope selection is governed by the (scaled) "free energy"

$$
\tilde{E}(h)=\int_{\Omega}\left[\frac{1}{4}\left(|\nabla h|^{2}-1\right)^{2}+\frac{1}{2}|\Delta h|^{2}\right] d x,
$$

where the first term selects the (scaled) mound slope 1 . This model predicts the exponent $\beta=1 / 3[15,16,21,23,28]$. Recently, Kohn and Yan [15] rigorously proved an averaged version of a one-sided bound for this one-third law.

For both of the models, we showed in [20] a nonlinear morphological instability in the rough-smooth-rough pattern that is experimentally observed [9] and the well-posedness of the corresponding initial-boundary-value problems. 
Setting $\varepsilon=1 / L$, we can re-scale the "energy" to get

$$
E(\hat{h})=E_{\varepsilon}(h) \quad \text { with } \quad h(x)=\hat{h}(\hat{x}) / L \quad \text { and } \quad \hat{x}=L x,
$$

where

$$
E_{\varepsilon}(h)=\int_{\Omega_{1}}\left[-\frac{1}{2} \log \left(1+|\nabla h|^{2}\right)+\frac{\varepsilon^{2}}{2}|\Delta h|^{2}\right] d x
$$

and $\Omega_{1}=(0,1)^{d}$ is the unit cube in $\mathbb{R}^{d}$. The related gradient flow, same as the scaled Eq. (1.1), is

$$
\partial_{t} h=-\nabla \cdot\left(\frac{\nabla h}{1+|\nabla h|^{2}}\right)-\varepsilon^{2} \Delta^{2} h .
$$

Our goal of this work is to understand the energetics, coarsening, and dynamic scaling of the interfacial dynamics in epitaxial growth without the slope selection, and justify rigorously the scaling laws predicted by the underlying model.

Our main results are as follows:

(1) For any solution $h$ of Eq. (1.1), we show for large time $t$ that

$$
\begin{aligned}
& w_{h}(t) \leq O\left(t^{1 / 2}\right), \\
& \left(f_{t_{0}}^{t} f_{\Omega}|\nabla h(x, \tau)|^{2} d x d \tau\right)^{1 / 2} \leq O\left(t^{1 / 4}\right), \\
& f_{t_{0}}^{t} E(h(\tau)) d \tau \geq O(-\log t) .
\end{aligned}
$$

See Section 2. All the bounds are independent of the dimension $d$ and the system size $L$. They are only one-sided. A two-sided bound is often not universally true. For instance, an upper bound for the "energy" like $E(h(t)) \leq O(-\log t)$ will not be true for a steady-state solution $h$.

Note that our basic bounds lead to the $O\left(-t^{1 / 2} \log t\right)$ lower bound for $E(h(t)) w_{h}(t)$. This is different from a constant lower bound for the same quantity in the slopeselection model, cf. [15];

(2) For any $\varepsilon>0$, we show that global minimizers of the scaled energy $E_{\varepsilon}$ defined in (1.8) exist. Here, a global minimizer is a height profile that has the least energy among the class of smooth and periodic height profiles. For small $\varepsilon>0$, we also show that

$$
\left\|\nabla^{m} h_{\varepsilon}\right\|_{L^{2}\left(\Omega_{1}\right)}=O\left(\frac{1}{\varepsilon}\right) \quad(m=0,1,2)
$$

for any energy minimizer of $E_{\varepsilon}$ and that

$$
\min _{h} E_{\varepsilon}(h) \sim \log \varepsilon .
$$


By a scaling argument, we can construct for each integer $j \geq 1$ an equilibrium solution $h_{j}$ of (1.1) with wavelength proportional to $L / j$, cf. Section 4 .

To better understand the variational properties of the model, we present in Section 3 some heuristic calculations in a one-dimensional setting of the rescaled energy (1.8) for a trial profile and of the local shape of an equilibrium solution of the rescaled equation (1.9);

(3) For any solution $h$ of Eq. (1.1), any $\xi \in \mathbb{R}$ with $1 / L<\xi<1$, and $t_{\xi}>0$ such that $E\left(h\left(t_{\xi}\right)\right)=-\log (\xi L)$, we show for large $t$ that

$$
\left(f_{t_{\xi}}^{t}\left[w_{h}(\tau)\right]^{2} d \tau\right)^{1 / 2} \geq O\left(\xi^{2} L^{2}\right) .
$$

Moreover, we show for any $t>t_{\xi}$ that in fact

$$
t \geq O\left((\xi L)^{\frac{4(\sigma-1)}{\sigma}}\right),
$$

where $\sigma=t / t_{\xi}$. See Section 5 .

Our approach is different from that in Kohn-Otto [14] and Kohn-Yan [15]: we do not need an isoperimetric inequality, since the $O\left(t^{1 / 2}\right)$ upper bound on the interface width can be easily obtained for the underlying model. Our analysis on the variational problem of minimizing the energy helps understand why the slope of mounds can grow unboundedly. It also helps determine a time scale for bounding the saturation value of the interface width and the corresponding saturation time.

There are several important issues that we have not been able to address and resolve in this work but we wish to further study:

(1) An upper bound for the characteristic lateral size of mounds $\lambda(t)$. For the slop-selection model, this size $\lambda(t)$ is of the same order as that of the height - the interface width. Thus, there is no need to do extra work to bound $\lambda(t)$. In general, a precise mathematical concept that describes the lateral size $\lambda(t)$ is needed;

(2) The optimality of bounds. For the slope-selection model, Ortiz, Repetto, and Shi [23] constructed a solution for the reduced dynamics that achieves the optimal bound. Can one have a similar construction for the underlying model without slope selection? The difficulty seems to lie in the fact that the energy (1.2) is not bounded below as the system becomes larger and larger;

(3) The limiting dynamics as $\varepsilon \rightarrow 0$. This is a non-trivial problem that is related to the singular perturbation, or regularization, of a conservation law, cf. Eq. (1.9). From the view point of energy minimization, one may try to calculate the related $\Gamma$-limit and 
gradient flow of such a limit to obtain the reduced dynamic law, just like what is done in [23] for the slope-selection model. But, again, the difficult is the unboundedness of the energy;

(4) The mathematical analysis and interpretation of the phase ordering method. This method is used to predict the underlying scaling laws (1.5) assuming a priori scaling laws with certain exponents [8]. Mathematically, we understand little about such a method. It will be interesting to explore such a method with the rigorous analysis presented in Kohn and Otto [14], Kohn and Yan [15], and this work;

(5) Rigorous analysis of evolution equation models for epitaxial growth that do not possess any Lyapunov functionals. Some good and important examples are the Kardar-ParisiZhang and Lai-Dar Sarma-Villain equations $[13,19,36]$. We expect that such analysis can be more difficult, and a new framework is needed.

\section{Bounds on the interface width, gradient, and energy}

Let $C_{\text {per }}^{\infty}(\bar{\Omega})$ be the set of all restrictions onto $\bar{\Omega}$ of all real-valued, $\bar{\Omega}$-periodic, $C^{\infty}$-functions on $\mathbb{R}^{d}$. For any integer $m \geq 0$, let $H_{p e r}^{m}(\Omega)$ be the closure of $C_{p e r}^{\infty}(\bar{\Omega})$ in the usual Sobolev space $W^{m, 2}(\Omega)[1,7]$. Let

$$
\mathcal{H}(\Omega)=\left\{h \in H_{\text {per }}^{2}(\Omega): f_{\Omega} h d x=0\right\} .
$$

It is clear that $\mathcal{H}(\Omega)$ is a closed subspace of $H_{\text {per }}^{2}(\Omega)$. Throughout the paper, we denote by $\|\cdot\|$ the $L^{2}$-norm for an underlying domain. We also write a function $u: \Omega \times[0, T] \rightarrow \mathbb{R}$ which is in a function space $X$ for each $t$ as a mapping $u=u(t):[0, T] \rightarrow X$.

Let $T>0$ and $h:[0, T] \rightarrow L^{2}(\Omega)$. The interface width for $h$ is defined for any $t \in[0, T]$ by

$$
w_{h}(t)=\sqrt{f_{\Omega}|h(x, t)-\bar{h}(t)|^{2} d x} \quad \text { with } \bar{h}(t)=f_{\Omega} h(x, t) d x .
$$

In particular,

$$
w_{h}(t)=\sqrt{f_{\Omega}|h(x, t)|^{2} d x} \quad \forall h \in \mathcal{H}(\Omega) .
$$

Theorem 2.1 (bounds on the interface width, gradient, and energy) Let $h(\cdot):[0, \infty) \rightarrow$ $\mathcal{H}(\Omega)$ be a weak solution of Eq. (1.1) on $(0, T)$ for any $T>0$ [20]. Let $t_{0} \geq 0$.

(1) An upper bound on the interface width. We have

$$
w_{h}(t) \leq \sqrt{2\left(t-t_{0}\right)+\left[w_{h}\left(t_{0}\right)\right]^{2}} \quad \forall t \geq t_{0} .
$$


(2) Upper bounds on the gradients. We have

$$
f_{t_{0}}^{t} f_{\Omega}|\Delta h(x, \tau)|^{2} d x d \tau \leq 1+\frac{\left[w_{h}\left(t_{0}\right)\right]^{2}}{2\left(t-t_{0}\right)} \quad \forall t>t_{0}
$$

and

$$
f_{t_{0}}^{t} f_{\Omega}|\nabla h(x, \tau)|^{2} d x d \tau \leq\left(1+\frac{\left[w_{h}\left(t_{0}\right)\right]^{2}}{2\left(t-t_{0}\right)}\right)^{1 / 2}\left(t+t_{0}+\left[w_{h}\left(t_{0}\right)\right]^{2}\right)^{1 / 2} \quad \forall t>t_{0} .
$$

(3) A lower bound on the energy. We have

$$
f_{t_{0}}^{t} E(h(\tau)) d t \geq-\frac{1}{2} \log (1+\sqrt{3 t}) \quad \forall t>t_{0}+\left[w_{h}\left(t_{0}\right)\right]^{2} .
$$

Note that, for large $t$, the bounds in (2.2), (2.4), and (2.5) are exactly those in (1.10), (1.11), and (1.12), respectively.

Proof of Theorem 2.1. (1) By the definition of a weak solution [20], we have (see [28])

$$
\frac{d}{d t}\left[w_{h}(t)\right]^{2}=2 f_{\Omega} h h_{t} d x=2 f_{\Omega}\left(\frac{|\nabla h|^{2}}{1+|\nabla h|^{2}}-|\Delta h|^{2}\right) d x \leq 2 \quad \forall t>0 .
$$

Thus, integrating from $t_{0}$ to $t>t_{0}$ and then taking the square root, we obtain (2.2).

(2) It follows from (2.6) that

$$
\frac{1}{2} \frac{d}{d t}\left[w_{h}(t)\right]^{2}+f_{\Omega}(\Delta h)^{2} d x=f_{\Omega} \frac{|\nabla h|^{2}}{1+|\nabla h|^{2}} d x \leq 1 .
$$

Thus, we have for any $t>t_{0}$ that

$$
f_{t_{0}}^{t} f_{\Omega}|\Delta h(x, \tau)|^{2} d x d \tau \leq 1+\frac{1}{t-t_{0}}\left(\frac{1}{2}\left[w_{h}\left(t_{0}\right)\right]^{2}-\frac{1}{2}\left[w_{h}(t)\right]^{2}\right),
$$

leading to $(2.3)$.

Now, it follows from an integration by parts, the Cauchy-Schwarz inequality, (2.2), and (2.3) that for any $t>t_{0}$

$$
\begin{aligned}
f_{t_{0}}^{t} f_{\Omega}|\nabla h(x, \tau)|^{2} d x d \tau & =f_{t_{0}}^{t} f_{\Omega}[-h(x, \tau)] \Delta h(x, \tau) d x d \tau \\
& \leq\left(f_{t_{0}}^{t} f_{\Omega}|h(x, \tau)|^{2} d x d \tau\right)^{1 / 2}\left(f_{t_{0}}^{t} f_{\Omega}|\Delta h(x, \tau)|^{2} d x d \tau\right)^{1 / 2}
\end{aligned}
$$




$$
\begin{aligned}
& \leq\left(f_{t_{0}}^{t}\left(2\left(\tau-t_{0}\right)+\left[w_{h}\left(t_{0}\right)\right]^{2}\right) d \tau\right)^{1 / 2}\left(1+\frac{\left[w_{h}\left(t_{0}\right)\right]^{2}}{2\left(t-t_{0}\right)}\right)^{1 / 2} \\
& \leq\left(t+t_{0}+\left[w_{h}\left(t_{0}\right)\right]^{2}\right)^{1 / 2}\left(1+\frac{\left[w_{h}\left(t_{0}\right)\right]^{2}}{2\left(t-t_{0}\right)}\right)^{1 / 2} .
\end{aligned}
$$

This proves (2.4).

(3) If $t>t_{0}+\left[w_{h}\left(t_{0}\right)\right]^{2}$, then

$$
\frac{\left[w_{h}\left(t_{0}\right)\right]^{2}}{2\left(t-t_{0}\right)} \leq \frac{1}{2} \quad \text { and } \quad t+t_{0}+\left[w_{h}\left(t_{0}\right)\right]^{2} \leq 2 t
$$

Since $-\log$ is a convex function, we obtain by Jensen's inequality, (2.4), and (2.7) that

$$
\begin{aligned}
f_{t_{0}}^{t} E(h(\tau)) d \tau & \geq-\frac{1}{2} \log \left(1+f_{t_{0}}^{t} f_{\Omega}|\nabla h(x, \tau)|^{2} d x d \tau\right) \\
& \geq-\frac{1}{2} \log \left(1+\left(1+\frac{\left[w_{h}\left(t_{0}\right)\right]^{2}}{2\left(t-t_{0}\right)}\right)^{1 / 2}\left(t+t_{0}+\left[w_{h}\left(t_{0}\right)\right]^{2}\right)^{1 / 2}\right) \\
& =-\frac{1}{2} \log (1+\sqrt{3 t})
\end{aligned}
$$

proving (2.5). Q.E.D.

\section{$3 \quad$ Heuristic calculations of energetics and equilibria}

In this section, we assume the space dimension is $d=1$ and consider the energy functional (1.8) and the related gradient flow (1.9). We first calculate simple height profiles that have low energies. We then determine the local shape of equilibria. Detailed one-dimensional analysis on the scaling laws can be found in [24].

\subsection{Energy of a trial profile}

Let $j \geq 1$ an integer. Divide the interval $[0,1]$ into $2 j$ small intervals of the same length $1 / 2 j$. Let $k>0$ and $\delta>0$ be real numbers with $2 \delta<1 / 2 j$. Define a trial function $h \in C^{1}[0,1]$ by

$$
h(x)= \begin{cases}k x & \text { if } 0 \leq x<\frac{1}{4 j}-\delta, \\ -\frac{k}{2 \delta}\left(x-\frac{1}{4 j}\right)^{2}+k\left(\frac{1}{4 j}-\frac{\delta}{2}\right) & \text { if } \frac{1}{4 j}-\delta \leq x<\frac{1}{4 j}+\delta, \\ -k\left(x-\frac{1}{2 j}\right) & \text { if } \frac{1}{4 j}+\delta \leq x<\frac{3}{4 j}-\delta, \\ \frac{k}{2 \delta}\left(x-\frac{3}{4 j}\right)^{2}-k\left(\frac{1}{4 j}-\frac{\delta}{2}\right) & \text { if } \frac{3}{4 j}-\delta \leq x<\frac{3}{4 j}+\delta, \\ k\left(x-\frac{1}{j}\right) & \text { if } \frac{3}{4 j}+\delta \leq x \leq \frac{1}{j},\end{cases}
$$


and

$$
h\left(x+\frac{1}{j}\right)=h(x) \quad \forall x \in\left(\frac{(i-1)}{j}, \frac{i}{j}\right], \quad i=2, \ldots, j .
$$

This function is quadratic in each "transition region" $\left(c_{i}-\delta, c_{i}+\delta\right)$ with $c_{i}=\frac{1}{2}[(i-1) / 2 j+i / 2 j]$ $(i=1, \ldots, 2 j)$ and linear with the slope $k$ or $-k$ elsewhere. See Figure 3.1.

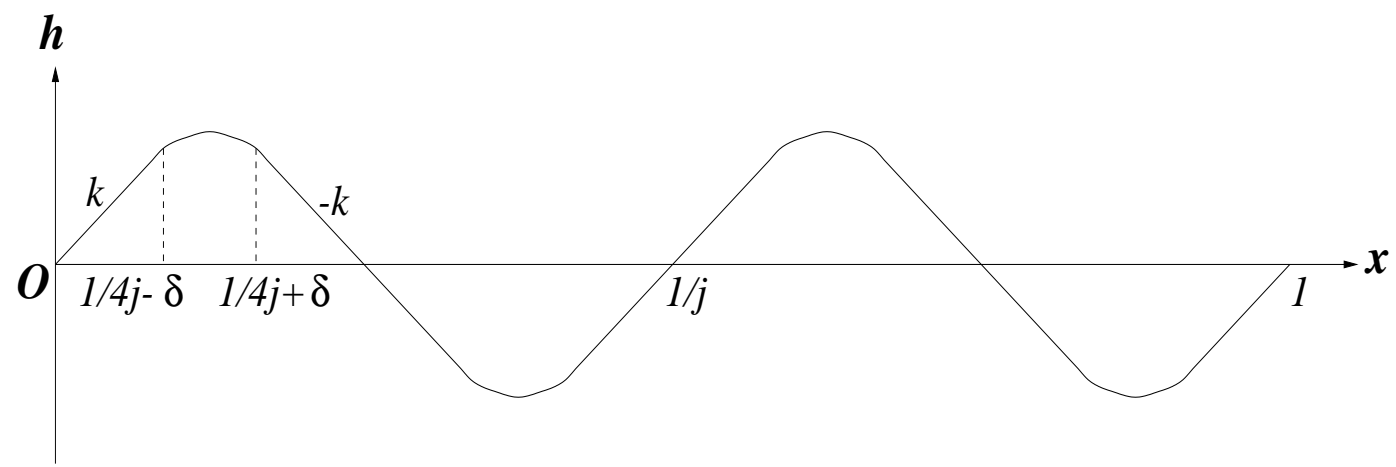

Figure 3.1. A trial height profile.

Straight forward calculations lead to

$$
\begin{aligned}
E_{\varepsilon}(h) & =\int_{0}^{1}\left[-\frac{1}{2} \log \left(1+h^{\prime 2}\right)+\frac{\varepsilon^{2}}{2} h^{\prime \prime 2}\right] d x \\
& =\left(\int_{\text {non-transition regions }}+\int_{\text {transition regions }}\right)\left[-\frac{1}{2} \log \left(1+h^{\prime 2}\right)+\frac{\varepsilon^{2}}{2} h^{\prime \prime 2}\right] d x \\
& =-\frac{1}{2} \log \left(1+k^{2}\right)+2 j \delta f(k)+\frac{2 j \varepsilon^{2} k^{2}}{\delta},
\end{aligned}
$$

where

$$
\begin{aligned}
f(k) & =\log \left(1+k^{2}\right)-\int_{0}^{1} \log \left(1+k^{2} s^{2}\right) d s \\
& =\int_{0}^{1} \log \left(\frac{1+k^{2}}{1+k^{2} s^{2}}\right) d s=\frac{1}{k} \int_{0}^{k} \log \left(\frac{1+k^{2}}{1+s^{2}}\right) d s .
\end{aligned}
$$

It is not difficult to see that $f$ increases on $(0, \infty)$ from 0 to 2 and $f(k) \sim 2 k^{2} / 3$ as $k \rightarrow 0^{+}$.

Fix $k$. The energy $E_{\varepsilon}(h)$ is minimized at $\delta=\varepsilon k / \sqrt{f(k)}$. At this value of $\delta$, the energy becomes

$$
E_{\varepsilon}(h)=-\frac{1}{2} \log \left(1+k^{2}\right)+4 j \varepsilon k \sqrt{f(k)} .
$$

With varying $k$, this is minimized at $k=g(k) /(j \varepsilon)$, where

$$
g(k)=\frac{k^{2} \sqrt{f(k)}}{2\left(1+k^{2}\right)\left[2 f(k)+k f^{\prime}(k)\right]}>0 .
$$


We have $g(k)>0$, since $f(k)>0$ and $f^{\prime}(k)>0$. Moreover, $g(k) \rightarrow \sqrt{2} / 8$ as $k \rightarrow \infty$, since

$$
k f^{\prime}(k)=\frac{2 k^{2}}{1+k^{2}} \int_{0}^{1} \frac{1-s^{2}}{1+k^{2} s^{2}} d s \rightarrow 0 \quad \text { as } k \rightarrow \infty .
$$

Thus, if $0<j \varepsilon \ll 1$, the optimal value of slope $k$ has the asymptotics

$$
k=O\left(\frac{1}{j \varepsilon}\right) \quad \text { for } k \gg 1 .
$$

With this $k$, the size of each transition region is

$$
\delta=\frac{g(k)}{j \sqrt{f(k)}}=O\left(\frac{1}{j}\right) \quad \text { for } k \gg 1,
$$

and the minimum energy is

$$
E_{\varepsilon}(h) \sim \log (j \varepsilon) \quad \text { as } \varepsilon \rightarrow 0^{+} .
$$

Our calculations indicate that the size of each transition region is of the same order as that of the base of a mound, and in particular, it is independent of $\varepsilon$. Moreover, in the large system limit $\varepsilon \rightarrow 0$, the mound slope of a global minimizer is proportional to the linear size of the underlying system. Finally, the minimum energy scales as $\log \varepsilon$ for small $\varepsilon>0$. All these properties are quite different from those of the slope selection model.

\subsection{The local shape of an equilibrium}

We now consider the one-dimensional steady-state equation

$$
\left(\frac{h^{\prime}}{1+h^{\prime 2}}\right)^{\prime}+\varepsilon^{2} h^{(4)}=0 \quad \text { on }(0,1)
$$

with the periodic boundary condition, cf. (1.9). We assume an equilibrium solution $h$ is a profile that consists of hills and valleys, similar to that shown in Figure 3.1. To understand the local shape of such an equilibrium solution, we assume without loss of generality that $h$ is an even and convex function on $[-a, a]$ for some real number $a>0$. We also assume that $h^{\prime}( \pm a)= \pm k$ for some constant $k>0$ and $h^{\prime \prime}( \pm a)=0$. See Figure 3.2.

Set $g=h^{\prime}$ and integrate both sides of Eq. (3.4) to get

$$
\frac{g}{1+g^{2}}+\varepsilon^{2} g^{\prime \prime}=c_{1}
$$

for some constant $c_{1}$. Clearly, $c_{1}=0$, since $g(0)=g^{\prime \prime}(0)=0$. Thus,

$$
\frac{g}{1+g^{2}}+\varepsilon^{2} g^{\prime \prime}=0
$$




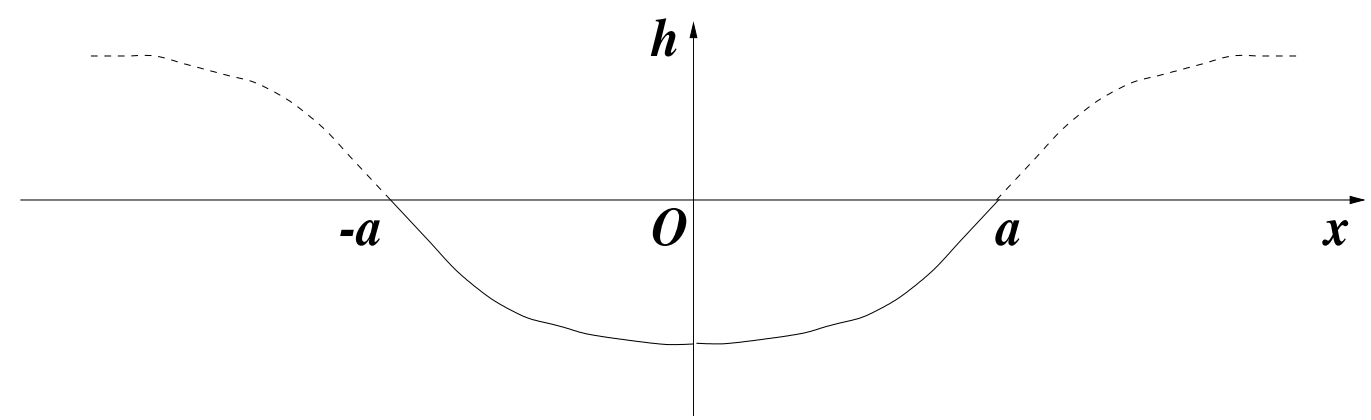

Figure 3.2. The local shape of an equilibrium solution.

Multiplying both sides of this equation by $2 g^{\prime}$ and integrate to get

$$
\log \left(1+g^{2}\right)+\varepsilon^{2} g^{\prime 2}=c_{2}
$$

where $c_{2}$ is a constant. Since $g(a)=k$ and $g^{\prime}(a)=0$, we obtain that $c_{2}=\log \left(1+k^{2}\right)$. Consequently,

$$
\log \left(\frac{1+g^{2}}{1+k^{2}}\right)+\varepsilon^{2} g^{\prime 2}=0
$$

Now solving Eq. (3.5) with $g^{\prime}=d g / d x$ and only considering $x \in[0, a]$, we get

$$
x=x(g)=\varepsilon \int_{0}^{g} \frac{d g}{\sqrt{\log \left(\frac{1+k^{2}}{1+g^{2}}\right)}} .
$$

Let $0<\sigma<1$. With the change of variables $g=k z$, we obtain that

$$
x(\sigma k)=\varepsilon \int_{0}^{\sigma k} \frac{d g}{\sqrt{\log \frac{1+k^{2}}{1+g^{2}}}}=\varepsilon \int_{0}^{\sigma} \frac{k d z}{\sqrt{\log \frac{1+k^{2}}{1+k^{2} z^{2}}}} .
$$

Here, $\sigma$ represents the ratio of the profile slope at the position $x$ and the far-field slope $k$. Clearly,

$$
x(\sigma k) \rightarrow 0 \quad \text { as } \sigma \rightarrow 0^{+} .
$$

Moreover, since $\left(1+k^{2}\right) /\left(1+k^{2} z^{2}\right) \leq 1 / z^{2}$ for $z \in(0,1)$, we get by changing the variable $y=1 / z$ that

$$
x(\sigma k) \geq k \varepsilon \int_{0}^{\sigma} \frac{d z}{\sqrt{\log \frac{1}{z^{2}}}}=k \varepsilon \int_{1 / \sigma}^{\infty} \frac{d y}{y^{2} \sqrt{2 \log y}} \rightarrow k \varepsilon \int_{1}^{\infty} \frac{d y}{y^{2} \sqrt{2 \log y}} \quad \text { as } \sigma \rightarrow 1 .
$$

The last integral on $[1, \infty)$ is finite and independent of $\varepsilon$ and $k$. It follows from (3.6) and (3.7) that the size of the transition region is $O(k \varepsilon)$. This agrees with (3.1) and (3.2). 
Multiplying both sides of Eq. (3.4) by $h$ and integrating by parts, we get

$$
\int_{0}^{1} \varepsilon^{2} h^{\prime \prime 2} d x=\int_{0}^{1} \frac{h^{\prime 2}}{1+h^{\prime 2}} d x \leq 1 .
$$

This, together with (3.5) which is generally satisfied by $g=h^{\prime}$ everywhere in $(0,1)$, leads to

$$
\begin{aligned}
E_{\varepsilon}(h) & =\int_{0}^{1}\left[-\frac{1}{2} \log \left(1+g^{2}\right)+\frac{\varepsilon^{2}}{2} g^{\prime 2}\right] d x \\
& =\int_{0}^{1}\left[-\frac{1}{2} \log \left(1+k^{2}\right)+\varepsilon^{2} g^{\prime 2}\right] d x \\
& \sim-\log k \quad \text { as } k \rightarrow \infty .
\end{aligned}
$$

This agrees with our previous calculations of the energetics, cf. (3.1) and (3.3).

\section{Energy minimization}

In this section, we study the variational problem of minimizing the scaled energy (1.8) defined on $\Omega_{1}=(0,1)^{d}$. Our main result is the following theorem which is a rigorous version of the basic properties we obtained from our heuristic calculations:

Theorem 4.1 (energy minimization) (1) For any $\varepsilon>0$, there exists $h_{\varepsilon} \in \mathcal{H}\left(\Omega_{1}\right)$ such that

$$
E_{\epsilon}\left(h_{\varepsilon}\right)=\min _{h \in \mathcal{H}\left(\Omega_{1}\right)} E_{\varepsilon}(h) .
$$

(2) Denote $e_{\varepsilon}=\min _{h \in \mathcal{H}\left(\Omega_{1}\right)} E_{\varepsilon}(h)$. There exists a constant $C_{1}>0$ such that

$$
e_{\varepsilon} \leq \log \varepsilon+C_{1} \quad \forall \varepsilon>0 .
$$

Moreover,

$$
e_{\varepsilon} \sim \log \varepsilon \quad \text { as } \varepsilon \rightarrow 0 .
$$

(3) There exist two constants $C_{2}>0$ and $C_{3}>0$ such that for any $\varepsilon \in\left(0, e^{-C_{1}} / \sqrt{2}\right)$ and any global minimizer $h_{\varepsilon} \in \mathcal{H}\left(\Omega_{1}\right)$ of $E_{\varepsilon}: \mathcal{H}\left(\Omega_{1}\right) \rightarrow \mathbb{R}$,

$$
\frac{C_{2}}{\varepsilon} \leq\left\|\nabla^{m} h_{\varepsilon}\right\| \leq \frac{C_{3}}{\varepsilon}, \quad m=0,1,2,
$$

where $\nabla^{0} h=h, \nabla^{1} h=\nabla h, \nabla^{2} h=\Delta h$, and $\|\cdot\|$ is the $L^{2}\left(\Omega_{1}\right)$-norm.

A direct consequence of the existence of global minimizers is the following result of the existence of a sequence of equilibrium solutions $h_{j}(j=1, \ldots)$ of the original equation (1.1) over $\Omega=(0, L)^{d}$ with each $h_{j}$ being $\bar{\Omega}_{L / j}$-periodic, where $\Omega_{L / j}=(0, L / j)^{d}$ : 
Corollary 4.1 For any integer $j \geq 1$, there exists $h_{j} \in \mathcal{H}(\Omega)$ that satisfies the following properties:

(1) The function $h_{j}$ is $\bar{\Omega}_{L / j}$-periodic. Moreover, if $h \in \mathcal{H}(\Omega)$ is $\bar{\Omega}_{L / j}$-periodic, then

$$
E\left(h_{j}\right) \leq E(h)
$$

In particular, for any integer $I \geq 0$, we have

$$
E\left(h_{2^{I}}\right) \geq \ldots \geq E\left(h_{2^{i}}\right) \geq E\left(h_{2^{i-1}}\right) \ldots \geq E\left(h_{1}\right)=\min _{h \in \mathcal{H}(\Omega)} E(h) .
$$

(2) The function $h_{j}$ is an equilibrium solution of Eq. (1.1), i.e.,

$$
\nabla \cdot\left(\frac{\nabla h_{j}}{1+\left|\nabla h_{j}\right|^{2}}\right)+\Delta^{2} h_{j}=0 \quad \text { in } \Omega
$$

Proof. Fix $j$. Let $\tilde{h}_{j} \in \mathcal{H}(\Omega)$ be a global energy minimizer of $E_{j}: \mathcal{H}(\Omega) \rightarrow \mathbb{R}$, where

$$
E_{j}(\tilde{h})=f_{\Omega}\left[-\frac{1}{2} \log \left(1+|\nabla \tilde{h}|^{2}\right)+\frac{j^{2}}{2}|\Delta \tilde{h}|^{2}\right] d x \quad \forall \tilde{h} \in \mathcal{H}(\Omega) .
$$

Now, define $h_{j}(x)=(1 / j) \tilde{h}_{j}(j x)$ for any $x \in \mathbb{R}^{d}$. One easily verifies that $h_{j}$ is $\bar{\Omega}_{L / j}$-periodic, and that (4.4) holds true, since for any $h \in \mathcal{H}(\Omega)$ that is $\bar{\Omega}_{L / j}$-periodic,

$$
E(h)=\frac{1}{j^{d}} E_{j}(\tilde{h}) \geq \frac{1}{j^{d}} E_{j}\left(\tilde{h}_{j}\right)=E\left(h_{j}\right)
$$

where $h(x)=(1 / j) \tilde{h}(j x)$ for any $x \in \mathbb{R}^{d}$. This proves (4.4) which in turn implies (4.5) directly. Part (1) is proved.

Since $\tilde{h}_{j} \in \mathcal{H}(\Omega)$ is a global energy minimizer of $E_{j}: \mathcal{H}(\Omega) \rightarrow \mathbb{R}$, it is a critical point, i.e., a weak solution of

$$
\nabla \cdot\left(\frac{\nabla h_{j}}{1+\left|\nabla h_{j}\right|^{2}}\right)+j^{2} \Delta^{2} h_{j}=0 \quad \text { in } \Omega \text {. }
$$

Equivalently,

$$
j^{2} \Delta\left(\Delta h_{j}\right)=-\nabla \cdot\left(\frac{\nabla h_{j}}{1+\left|\nabla h_{j}\right|^{2}}\right) \quad \text { in } \Omega .
$$

Thus, by a simple argument using the regularity theory of elliptic equations and the fact that $h_{j}$ is periodic, we see that $h_{j}$ is smooth and satisfies Eq. (4.7) pointwise. Consequently, Eq. (4.6) follows from the scaling $h_{j}(x)=(1 / j) \tilde{h}_{j}(j x)$. Q.E.D.

To prove Theorem 4.1, we need several lemmas. For the first lemma, see Figure 4.1. 
Lemma 4.1 (1) If $\mu \geq 1$, then

$$
\log (1+s)<\mu s \quad \forall s>0 .
$$

(2) If $\mu \in(0,1)$, then there exists a unique $s_{\mu} \in(0, \infty)$ such that

$$
\log \left(1+s_{\mu}\right)=\mu s_{\mu},
$$

and that

$$
\begin{array}{ll}
\log (1+s)>\mu s & \text { if } s \in\left(0, s_{\mu}\right), \\
\log (1+s)<\mu s & \text { if } s \in\left(s_{\mu}, \infty\right) .
\end{array}
$$

(3) As a function of $\mu, s_{\mu}$ defined above increases from 0 to $\infty$ as $\mu$ decreases from 1 to 0. Moreover,

$$
\lim _{\mu \rightarrow 0^{+}} \frac{\log \left(1+s_{\mu}\right)}{\log \frac{1}{\mu}}=1 .
$$

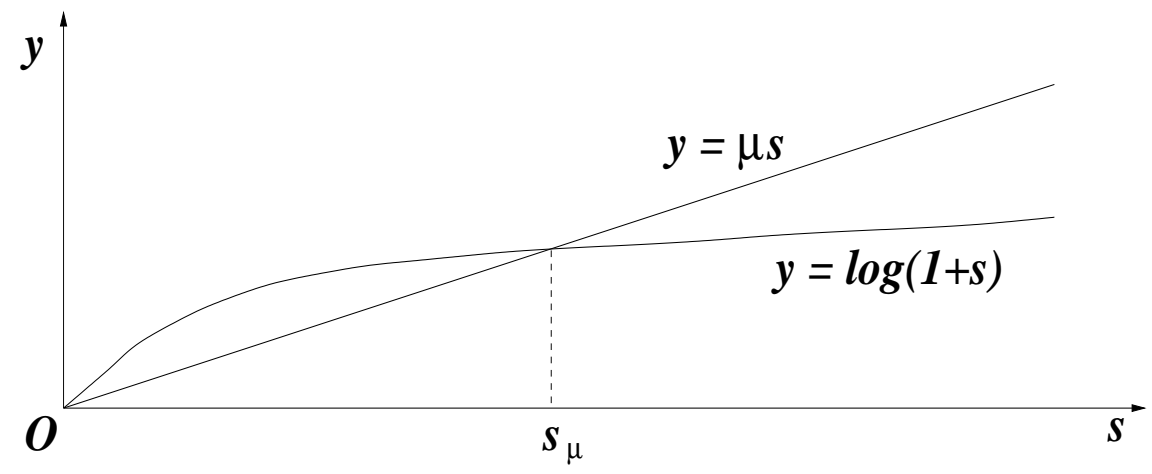

Figure 4.1. The meaning of $s_{\mu}$ in Lemma 4.1

Proof. (1) This follows from the fact that

$$
1+s<e^{s} \leq e^{\mu s} \quad \forall s>0 .
$$

(2) Fix $\mu \in(0,1)$. Let $f(s)=\log (1+s)-\mu s(s>-1)$ and $s_{0}=1 / \mu-1>0$. Then, $f^{\prime}\left(s_{0}\right)=0$. We have that $f(s)>0$ on $\left(0, s_{0}\right)$, since $f^{\prime}(s)>0$ on $\left(0, s_{0}\right)$ and $f(0)=0$. Similarly, $f^{\prime}(s)<0$ on $\left(s_{0}, \infty\right)$, and $f\left(s_{0}\right)=\mu-\log \mu-1$. This is a decreasing function of $\mu \in(0,1)$, since the derivative with respect to $\mu \in(0,1)$ is negative. Moreover, its value at $\mu=1$ is 0 . Thus, $f\left(s_{0}\right)>0$. Also, $f(s) \rightarrow-\infty$ as $s \rightarrow \infty$. Thus, there exists a unique $s_{\mu} \in\left(s_{0}, \infty\right)$ that satisfies (4.9) and (4.10).

(3) Notice that $\mu=s^{-1} \log (1+s)$ defines a continuously differentiable function for $s \in$ $(0, \infty)$ with $\mu^{\prime}(s)<0$, and $\mu(s) \rightarrow 1$ as $s \rightarrow 0^{+}$and $\mu(s) \rightarrow 0$ as $s \rightarrow \infty$. Thus, by the inverse function theorem, $s=s(\mu)$ defines a function of $\mu \in(0,1)$. Obviously, $s(\mu)=s_{\mu}$ as 
defined in (4.9). Moreover, since $s^{\prime}(\mu)=1 / \mu^{\prime}(s)<0, s_{\mu}$ increases from 0 to $\infty$ as $\mu$ decreases from 1 to 0 . Finally, taking logarithmic function of both sides of (4.9) and dividing them further by $\log s_{\mu}$, we get

$$
\frac{\log \log \left(1+s_{\mu}\right)}{\log s_{\mu}}=\frac{\log \mu}{\log s_{\mu}}+1=\frac{\log \mu}{\log \left(1+s_{\mu}\right)} \cdot \frac{\log \left(1+s_{\mu}\right)}{\log s_{\mu}}+1 .
$$

Since $s_{\mu} \rightarrow \infty$ as $\mu \rightarrow 0^{+}$,

$$
\frac{\log \log \left(1+s_{\mu}\right)}{\log s_{\mu}}=\frac{\log \log \left(1+s_{\mu}\right)}{\log \left(1+s_{\mu}\right)} \cdot \frac{\log \left(1+s_{\mu}\right)}{\log s_{\mu}} \rightarrow 0 \quad \text { as } \mu \rightarrow 0^{+} .
$$

Now, (4.11) follows from (4.12) and (4.12). Q.E.D.

We recall that $\|\Delta h\|$ is exactly the semi-norm $|h|_{H^{2}\left(\Omega_{1}\right)}$ for any $h \in H_{p e r}^{2}\left(\Omega_{1}\right)$, i.e.,

$$
\|\Delta h\|^{2}=\sum_{i, j=1}^{d}\left\|\partial_{x_{i} x_{j}} h\right\|^{2} \quad \forall h \in H_{p e r}^{2}\left(\Omega_{1}\right),
$$

cf. (1.3). We also recall the following Poincaré inequalities for the unit cell $\Omega_{1}=(0,1)^{d}[7]$ :

$$
\begin{aligned}
& \|h\| \leq C_{4}\|\nabla h\| \quad \forall h \in \mathcal{H}\left(\Omega_{1}\right), \\
& \|\nabla h\| \leq C_{5}\|\Delta h\| \quad \forall h \in \mathcal{H}\left(\Omega_{1}\right),
\end{aligned}
$$

where $C_{4}>0$ and $C_{5}>0$ are constants. The first inequality follows from the fact that any $h \in \mathcal{H}\left(\Omega_{1}\right)$ has zero mean. The second inequality follows from the fact that the mean of any first-order partial derivative of $h \in \mathcal{H}\left(\Omega_{1}\right)$ over $\Omega_{1}$ vanishes.

Lemma 4.2 (lower bound) Let $\varepsilon>0$ and $\mu=\varepsilon^{2} /\left(2 C_{5}^{2}\right)>0$. Let $s(\varepsilon)=0$ if $\mu \geq 1$ and $s(\varepsilon)=s_{\mu} \in(0, \infty)$, as defined in Lemma 4.1, if $0<\mu<1$. We have

$$
E_{\varepsilon}(h) \geq-\frac{1}{2} \log (1+s(\varepsilon))+\frac{\varepsilon^{2}}{4} \int_{\Omega_{1}}(\Delta h)^{2} d x \quad \forall h \in \mathcal{H}\left(\Omega_{1}\right) .
$$

Proof. Fix $h \in \mathcal{H}\left(\Omega_{1}\right)$. We have by Lemma 4.1 and the Poincaré inequality (4.16) that

$$
\begin{aligned}
E_{\varepsilon}(h)=- & \frac{1}{2} \int_{\left\{x \in \Omega_{1}:|\nabla h|^{2} \leq s(\varepsilon)\right\}} \log \left(1+|\nabla h|^{2}\right) d x \\
& -\frac{1}{2} \int_{\left\{x \in \Omega_{1}:|\nabla h|^{2}>s(\varepsilon)\right\}} \log \left(1+|\nabla h|^{2}\right) d x+\frac{\varepsilon^{2}}{2} \int_{\Omega_{1}}(\Delta h)^{2} d x \\
\geq & -\frac{1}{2} \log (1+s(\varepsilon))-\frac{\varepsilon^{2}}{4 C_{5}^{2}} \int_{\Omega_{1}}|\nabla h|^{2} d x+\frac{\varepsilon^{2}}{2} \int_{\Omega_{1}}(\Delta h)^{2} d x \\
\geq & -\frac{1}{2} \log (1+s(\varepsilon))+\frac{\varepsilon^{2}}{4} \int_{\Omega_{1}}(\Delta h)^{2} d x,
\end{aligned}
$$

as desired. Q.E.D. 
Lemma 4.3 (upper bound) For each $\varepsilon>0$, there exists $\tilde{h}_{\varepsilon} \in \mathcal{H}\left(\Omega_{1}\right)$ such that

$$
E_{\varepsilon}\left(\tilde{h}_{\varepsilon}\right) \leq \log \varepsilon+C_{6} \quad \forall \varepsilon>0,
$$

where $C_{6}>0$ is a constant independent of $\varepsilon$.

Proof. To better illustrate the idea, we prove the result for the case $d=2$. The general case can be treated similarly.

Define as before a 1-periodic, $C^{1}$-function $\phi_{\varepsilon}: \mathbb{R} \rightarrow \mathbb{R}$ by

$$
\phi_{\varepsilon}(s)= \begin{cases}k s & \text { if } 0 \leq s<\frac{1}{8} \\ -4 k\left(s-\frac{1}{4}\right)^{2}+\frac{3 k}{16} & \text { if } \frac{1}{8} \leq s<\frac{3}{8} \\ -k\left(s-\frac{1}{2}\right)^{2} & \text { if } \frac{3}{8} \leq s<\frac{5}{8} \\ 4 k\left(s-\frac{3}{4}\right)^{2}-\frac{3 k}{16} & \text { if } \frac{5}{8} \leq s<\frac{7}{8} \\ k(s-1) & \text { if } \frac{7}{8} \leq s \leq 1\end{cases}
$$

where $k=1 / \sqrt{\varepsilon}$. Note that we choose the slope $k$ to be proportional to $1 / \sqrt{\varepsilon}$ not $1 / \varepsilon$, cf. (3.1). This is because the constructed profile will be a product of two such one-dimensional trial functions. Note also that we choose the size of a "transition region" to be $\delta=1 / 8$. It is easy to verify that

$$
\begin{gathered}
\int_{0}^{1} \phi(s) d s=0, \\
\left|\phi_{\varepsilon}(s)\right| \geq \frac{k}{8} \quad \forall s \in\left[\frac{1}{8}, \frac{3}{8}\right] \cup\left[\frac{5}{8}, \frac{7}{8}\right],
\end{gathered}
$$

and

$$
\left|\phi_{\varepsilon}(s)\right| \leq \frac{3 k}{16}, \quad\left|\phi_{\varepsilon}^{\prime}(s)\right| \leq k, \quad\left|\phi_{\varepsilon}^{\prime \prime}(s)\right| \leq 8 k, \quad \text { a.e. } s \in \mathbb{R} .
$$

Define

$$
\tilde{h}_{\varepsilon}(x)=\phi_{\varepsilon}\left(x_{1}\right) \phi_{\varepsilon}\left(x_{2}\right) \quad \forall x=\left(x_{1}, x_{2}\right) \in \mathbb{R}^{2} .
$$

Clearly, $\tilde{h}_{\varepsilon} \in \mathcal{H}\left(\Omega_{1}\right)$.

We now calculate and estimate the energy

$$
E_{\varepsilon}\left(\tilde{h}_{\varepsilon}\right)=\int_{\Omega_{1}}\left[-\frac{1}{2} \log \left(1+\left|\nabla \tilde{h}_{\varepsilon}\right|^{2}\right)+\frac{\varepsilon^{2}}{2}\left(\Delta \tilde{h}_{\varepsilon}\right)^{2}\right] d x .
$$

The second term in the energy $E_{\varepsilon}\left(\tilde{h}_{\varepsilon}\right)$ is easy to bound by (4.18) and the fact that $k=1 / \sqrt{\varepsilon}$ :

$$
\begin{aligned}
\frac{\varepsilon^{2}}{2} \int_{\Omega_{1}}\left(\Delta \tilde{h}_{\varepsilon}\right)^{2} d x & =\frac{\varepsilon^{2}}{2} \int_{\Omega_{1}}\left[\phi_{\varepsilon}^{\prime \prime}\left(x_{1}\right) \phi_{\varepsilon}\left(x_{2}\right)+\phi_{\varepsilon}\left(x_{1}\right) \phi_{\varepsilon}^{\prime \prime}\left(x_{2}\right)\right]^{2} d x \\
& \leq \varepsilon^{2} \int_{\Omega_{1}}\left[\left|\phi_{\varepsilon}^{\prime \prime}\left(x_{1}\right)\right|^{2}\left|\phi_{\varepsilon}\left(x_{2}\right)\right|^{2}+\left|\phi_{\varepsilon}\left(x_{1}\right)\right|^{2}\left|\phi_{\varepsilon}^{\prime \prime}\left(x_{2}\right)\right|^{2}\right] d x
\end{aligned}
$$




$$
\leq \frac{9}{2}
$$

For the first term in the energy $E_{\varepsilon}\left(\tilde{h}_{\varepsilon}\right)$, we have by the symmetry and (4.17) that

$$
\begin{aligned}
& \int_{\Omega_{1}}-\frac{1}{2} \log \left(1+\left|\nabla \tilde{h}_{\varepsilon}\right|^{2}\right) d x \\
& =-8 \int_{(0,1 / 4) \times(0,1 / 4)} \log \left(1+\left|\nabla \tilde{h}_{\varepsilon}\right|^{2}\right) d x \\
& =-8 \int_{(0,1 / 4) \times(0,1 / 4)} \log \left[1+\left|\phi_{\varepsilon}^{\prime}\left(x_{1}\right)\right|^{2}\left|\phi_{\varepsilon}\left(x_{2}\right)\right|^{2}+\left|\phi_{\varepsilon}\left(x_{1}\right)\right|^{2}\left|\phi_{\varepsilon}^{\prime}\left(x_{2}\right)\right|^{2}\right] d x \\
& =-8 \int_{(0,1 / 8) \times(0,1 / 8)} \log \left[1+k^{4}\left(x_{1}^{2}+x_{2}^{2}\right)\right] d x \\
& -8 \int_{(1 / 8,1 / 4) \times(0,1 / 8)} \log \left[1+\left|\phi_{\varepsilon}^{\prime}\left(x_{1}\right)\right|^{2}\left|\phi_{\varepsilon}\left(x_{2}\right)\right|^{2}+k^{2}\left|\phi_{\varepsilon}\left(x_{1}\right)\right|^{2}\right] d x \\
& -8 \int_{(0,1 / 8) \times(1 / 8,1 / 4)} \log \left[1+k^{2}\left|\phi_{\varepsilon}\left(x_{2}\right)\right|^{2}+\left|\phi_{\varepsilon}\left(x_{1}\right)\right|^{2}\left|\phi_{\varepsilon}^{\prime}\left(x_{2}\right)\right|^{2}\right] d x \\
& -8 \int_{(1 / 8,1 / 4) \times(1 / 8,1 / 4)} \log \left[1+\left|\phi_{\varepsilon}^{\prime}\left(x_{1}\right)\right|^{2}\left|\phi_{\varepsilon}\left(x_{2}\right)\right|^{2}+\left|\phi_{\varepsilon}\left(x_{1}\right)\right|^{2}\left|\phi_{\varepsilon}^{\prime}\left(x_{2}\right)\right|^{2}\right] d x \\
& \leq-8 \int_{(0,1 / 8) \times(0,1 / 8)} \log \left[k^{4}\left(x_{1}^{2}+x_{2}^{2}\right)\right] d x \\
& -8 \int_{(1 / 8,1 / 4) \times(0,1 / 8)} \log \left[k^{2}\left|\phi_{\varepsilon}\left(x_{1}\right)\right|^{2}\right] d x \\
& -8 \int_{(0,1 / 8) \times(1 / 8,1 / 4)} \log \left[k^{2}\left|\phi_{\varepsilon}\left(x_{2}\right)\right|^{2}\right] d x \\
& -8 \int_{(1 / 8,1 / 4) \times(1 / 8,1 / 4)} \log \left[\left|\phi_{\varepsilon}^{\prime}\left(x_{1}\right)\right|^{2}\left|\phi_{\varepsilon}\left(x_{2}\right)\right|^{2}+\left|\phi_{\varepsilon}\left(x_{1}\right)\right|^{2}\left|\phi_{\varepsilon}^{\prime}\left(x_{2}\right)\right|^{2}\right] d x \\
& \leq-\frac{1}{8} \log k^{4}-8 \int_{(0,1 / 8) \times(0,1 / 8)} \log \left(x_{1}^{2}+x_{2}^{2}\right) d x \\
& -\frac{1}{8} \log \left(\frac{k^{4}}{64}\right)-\frac{1}{8} \log \left(\frac{k^{4}}{64}\right) \\
& -8 \int_{(1 / 8,1 / 4) \times(1 / 8,1 / 4)} \log \left\{\left[8 k\left(x_{1}-\frac{1}{4}\right)\right]^{2}\left(\frac{k}{8}\right)^{2}\right. \\
& \left.+\left(\frac{k}{8}\right)^{2}\left[8 k\left(x_{2}-\frac{1}{4}\right)\right]^{2}\right\} d x \\
& =\log \varepsilon+C_{7},
\end{aligned}
$$


where

$$
\begin{aligned}
C_{7}= & \frac{3}{2} \log 2-8 \int_{(0,1 / 8) \times(0,1 / 8)} \log \left(x_{1}^{2}+x_{2}^{2}\right) d x \\
& -8 \int_{(1 / 8,1 / 4) \times(1 / 8,1 / 4)} \log \left[\left(x_{1}-\frac{1}{4}\right)^{2}+\left(x_{2}-\frac{1}{4}\right)^{2}\right] d x
\end{aligned}
$$

is a finite number. This, together with (4.19), leads to the desired estimate with $C_{6}=$ $C_{7}+9 / 2$. Q.E.D.

Lemma 4.4 (equi-distribution of energy) If $h_{\varepsilon} \in H_{\text {per }}^{2}\left(\Omega_{1}\right)$ is a critical point of $E_{\varepsilon}$ : $H_{p e r}^{2}\left(\Omega_{1}\right) \rightarrow \mathbb{R}$, then

$$
\int_{\Omega} \varepsilon^{2}\left(\Delta h_{\varepsilon}\right)^{2} d x=\int_{\Omega} \frac{\left|\nabla h_{\varepsilon}\right|^{2}}{1+\left|\nabla h_{\varepsilon}\right|^{2}} d x
$$

Proof. If $h_{\varepsilon} \in H_{\text {per }}^{2}\left(\Omega_{1}\right)$ is a critical point of $E_{\varepsilon}: H_{p e r}^{2}\left(\Omega_{1}\right) \rightarrow \mathbb{R}$, then

$$
\delta E_{\varepsilon}\left(h_{\varepsilon}\right)(g)=\int_{\Omega}\left(-\frac{\nabla h_{\varepsilon} \cdot \nabla g}{1+\left|\nabla h_{\varepsilon}\right|^{2}}+\varepsilon^{2} \Delta h_{\varepsilon} \Delta g\right) d x=0, \quad \forall g \in H_{p e r}^{2}\left(\Omega_{1}\right) .
$$

Choosing $g=h_{\varepsilon}$, we obtain the desired identity. Q.E.D.

\section{Proof of Theorem 4.1.}

(1) Fix $\varepsilon>0$. By Lemma 4.2, we have $e_{\varepsilon}=\inf _{h \in \mathcal{H}\left(\Omega_{1}\right)} E_{\varepsilon}(h)>-\infty$. Let $\left\{h_{j}\right\}_{j=1}^{\infty}$ be an infimizing sequence of $E_{\varepsilon}: \mathcal{H}\left(\Omega_{1}\right) \rightarrow \mathbb{R}$. It follows from Lemma 4.2, the Poincaré inequalities (4.15) and (4.16), and the identity (4.14) that $\left\{h_{j}\right\}_{j=1}^{\infty}$ is bounded in $H_{p e r}^{2}\left(\Omega_{1}\right)$. Thus, up to a subsequence, $h_{j} \rightarrow h_{\varepsilon}$ in $H^{2}\left(\Omega_{1}\right)$ for some $h_{\varepsilon} \in H_{p e r}^{2}\left(\Omega_{1}\right)$. In particular, $\Delta h_{j} \rightarrow \Delta h_{\varepsilon}$ in $L^{2}\left(\Omega_{1}\right)$ and, up to a further subsequence if necessary, $h_{j} \rightarrow h_{\varepsilon}$ in $H^{1}\left(\Omega_{1}\right)$ as $j \rightarrow \infty$. Thus, $h_{\varepsilon} \in \mathcal{H}\left(\Omega_{1}\right)$. Moreover, by (4.8) with $\mu=1$ and the Cauchy-Schwarz inequality,

$$
\begin{aligned}
\mid \int_{\Omega_{1}} & {\left[\log \left(1+\left|\nabla h_{j}\right|^{2}\right)-\log \left(1+\left|\nabla h_{\varepsilon}\right|^{2}\right)\right] d x \mid } \\
& =\left|\int_{\Omega_{1}} \log \left(1+\frac{\left|\nabla h_{j}\right|^{2}-\left|\nabla h_{\varepsilon}\right|^{2}}{1+\left|\nabla h_{\varepsilon}\right|^{2}}\right) d x\right| \\
& \leq \int_{\Omega_{1}} \log \left(1+\left|\frac{\left|\nabla h_{j}\right|^{2}-\left|\nabla h_{\varepsilon}\right|^{2}}{1+\left|\nabla h_{\varepsilon}\right|^{2}}\right|\right) d x \\
& \leq \int_{\Omega_{1}}\left|\frac{\left|\nabla h_{j}\right|^{2}-\left|\nabla h_{\varepsilon}\right|^{2}}{1+\left|\nabla h_{\varepsilon}\right|^{2}}\right| d x \\
& \leq\left(\left\|\nabla h_{j}\right\|+\left\|\nabla h_{\varepsilon}\right\|\right)|| \nabla h_{j}-\nabla h_{\varepsilon} \| \\
& \rightarrow 0 \quad \text { as } j \rightarrow \infty .
\end{aligned}
$$


Further, since for each $j \geq 1,\left(\Delta h_{j}\right)^{2}+\left(\Delta h_{\varepsilon}\right)^{2} \geq 2 \Delta h_{j} \Delta h_{\varepsilon}$ in $\Omega_{1}$, we have

$$
\liminf _{j \rightarrow \infty} \int_{\Omega_{1}}\left(\Delta h_{j}\right)^{2} d x \geq \liminf _{j \rightarrow \infty}\left[2 \int_{\Omega_{1}} \Delta h_{j} \Delta h_{\varepsilon} d x-\int_{\Omega_{1}}\left(\Delta h_{\varepsilon}\right)^{2} d x\right]=\int_{\Omega_{1}}\left(\Delta h_{\varepsilon}\right)^{2} d x .
$$

Therefore,

$$
e_{\varepsilon}=\liminf _{j \rightarrow \infty} E_{\varepsilon}\left(h_{j}\right) \geq \int_{\Omega_{1}}\left[-\frac{1}{2} \log \left(1+\left|\nabla h_{\varepsilon}\right|^{2}\right)+\frac{\varepsilon^{2}}{2}\left(\Delta h_{\varepsilon}\right)^{2}\right] d x=E_{\varepsilon}\left(h_{\varepsilon}\right) \geq e_{\varepsilon} .
$$

This implies that $h_{\varepsilon}$ is a global minimizer of $E_{\varepsilon}$ in $\mathcal{H}\left(\Omega_{1}\right)$.

(2) The estimate (4.1) follows from Lemma 4.3 with $C_{1}=C_{6}$. It follows from Lemma 4.2, the definition of $s(\varepsilon)$ (cf. Lemma 4.2), and (4.11) in Lemma 4.1 that

$$
\liminf _{\varepsilon \rightarrow 0^{+}} \frac{e_{\varepsilon}}{\log \varepsilon}=\liminf _{\varepsilon \rightarrow 0^{+}} \frac{E_{\varepsilon}\left(h_{\varepsilon}\right)}{\log \varepsilon} \geq \liminf _{\varepsilon \rightarrow 0^{+}} \frac{-\frac{1}{2} \log (1+s(\varepsilon))}{\log \varepsilon}=1 .
$$

By Lemma 4.3,

$$
\limsup _{\varepsilon \rightarrow 0^{+}} \frac{e_{\varepsilon}}{\log \varepsilon} \leq \limsup _{\varepsilon \rightarrow 0^{+}} \frac{\log \varepsilon+C_{6}}{\log \varepsilon}=1
$$

Now, the desired asymptotics (4.2) follows from (4.20) and (4.21).

(3) Let $h_{\varepsilon} \in \mathcal{H}\left(\Omega_{1}\right)$ be a global minimizer of $E_{\varepsilon}: \mathcal{H}\left(\Omega_{1}\right) \rightarrow \mathbb{R}$. Clearly, $h_{\varepsilon}$ is also a global minimizer of $E_{\varepsilon}: H_{p e r}^{2}\left(\Omega_{1}\right) \rightarrow \mathbb{R}$. Thus, $h_{\varepsilon}$ is a critical point of $E_{\varepsilon}: H_{p e r}^{2}\left(\Omega_{1}\right) \rightarrow \mathbb{R}$. Consequently, we have by Lemma 4.4 that

$$
\varepsilon^{2} \int_{\Omega_{1}}\left(\Delta h_{\varepsilon}\right)^{2} d x=\int_{\Omega_{2}} \frac{\left|\nabla h_{\varepsilon}\right|^{2}}{1+\left|\nabla h_{\varepsilon}\right|^{2}} \leq 1
$$

Noting that the negative logarithmic function is convex, we have by Jensen's inequality that

$$
e_{\varepsilon}=E_{\varepsilon}\left(h_{\varepsilon}\right) \geq-\frac{1}{2} \log \left(1+\int_{\Omega_{1}}\left|\nabla h_{\varepsilon}\right|^{2} d x\right) .
$$

This, together with the upper bound (4.1), leads to

$$
\log \varepsilon+C_{1} \geq-\frac{1}{2} \log \left(1+\int_{\Omega_{1}}\left|\nabla h_{\varepsilon}\right|^{2} d x\right) .
$$

Hence,

$$
\int_{\Omega_{1}}\left|\nabla h_{\varepsilon}\right|^{2} d x \geq \frac{e^{-2 C_{1}}}{2 \varepsilon^{2}} \quad \text { if } \varepsilon \in\left(0, \frac{1}{\sqrt{2}} e^{-C_{1}}\right) .
$$

From (4.23), we have by an integration by parts, the Cauchy-Schwarz inequality, and (4.22) that

$$
\frac{e^{-2 C_{1}}}{2 \varepsilon^{2}} \leq \int_{\Omega_{1}}\left|\nabla h_{\varepsilon}\right|^{2} d x=\int_{\Omega_{1}}\left(-h_{\varepsilon}\right) \Delta h_{\varepsilon} d x
$$




$$
\leq\left(\int_{\Omega_{1}}\left|h_{\varepsilon}\right|^{2} d x\right)^{1 / 2}\left(\int_{\Omega_{1}}\left|\Delta h_{\varepsilon}\right|^{2} d x\right)^{1 / 2} \leq \frac{1}{\varepsilon}\left(\int_{\Omega_{1}}\left|h_{\varepsilon}\right|^{2} d x\right)^{1 / 2},
$$

leading to

$$
\int_{\Omega_{1}}\left|h_{\varepsilon}\right|^{2} d x \geq \frac{e^{-4 C_{1}}}{4 \varepsilon^{2}}
$$

Now all the estimates in (4.3) follow from (4.22), (4.24), the Poincaré inequalities (4.15) and (4.16), and the equivalence of norms (4.14). Q.E.D.

\section{Bounds on the saturation interface width and satu- ration time}

We now consider the free energy (1.2) that is defined with $\Omega=(0, L)^{d}$. By Theorem 4.1 and the change of variables in (1.7), we have that

$$
E_{L}:=\min _{h \in \mathcal{H}(\Omega)} E(h) \sim-\log L \quad \text { as } L \rightarrow \infty,
$$

and for $L \geq \sqrt{2} e^{C_{1}}$ that

$$
C_{2} L^{4-2 m} \leq f_{\Omega}\left|\nabla^{m} h_{L}\right|^{2} d x \leq C_{3} L^{4-2 m}, \quad m=0,1,2,
$$

where $h_{L} \in \mathcal{H}(\Omega)$ is any minimizer of $E: \mathcal{H}(\Omega) \rightarrow \mathbb{R}$, and $C_{2}$ and $C_{3}$ are the same constants as in (4.3).

It is reasonable to think that the profile will be near a global minimizer after the saturation of the interface width. Recall from Theorem 2.1 that the interface width is bounded above by $O\left(t^{1 / 2}\right)$. This, together with (5.1) with $m=0$, then sets the saturation time $t_{s}=O\left(L^{4}\right)$, and hence the saturation interface width $w_{s}(L)=O\left(t_{s}^{1 / 2}\right)=O\left(L^{2}\right)$. These are exactly the predicted scaling laws, cf. (1.5). The following result is a rigorous justification of some forms of these scaling laws.

Theorem 5.1 Let $h(\cdot):[0, \infty) \rightarrow \mathcal{H}(\Omega)$ be a weak solution of Eq. (1.1) on $(0, T)$ for any $T>0$. Let $L>\sqrt{2}$ and $\xi \in(\sqrt{2} / L, 1)$. Let $t_{\xi}>0$ be such that

$$
E\left(h\left(t_{\xi}\right)\right)=-\log (\xi L) .
$$

(1) If $t \geq 2 t_{\xi}+(1 / 2)\left[w_{h}(0)\right]^{2}$, then

$$
\left(f_{t_{\xi}}^{t}\left[w_{h}(\tau)\right]^{2} d \tau\right)^{1 / 2} \geq \frac{\xi^{2}}{\sqrt{8}} L^{2} .
$$


(2) If $t>\max \left(t_{\xi}, 1 / 3,\left[w_{h}(0)\right]^{2}\right)$, then in fact

$$
t \geq \frac{1}{12} e^{-\frac{4}{\sigma} E(h(0))}(\xi L)^{\frac{4(\sigma-1)}{\sigma}},
$$

where $\sigma=t / t_{\xi}$.

We remark that the time $t_{\xi}$ approximates the saturation time. In general, $t_{\xi}$ is larger when the parameter $\xi$ is closer to 1. The inequality (5.3) gives a lower bound, in an averaged version, for the saturation interface width. The inequality (5.4) is an asymptotic, lower bound for the saturation time: as $\xi$ is closer to 1 and $\sigma$ becomes very large, the bound becomes $O\left(L^{4}\right)$. The assumption for this lower bound is that the time $t$ is larger than $t_{\xi}$ which is the time at which the energy of a profile $h$ reaches nearly the minimum value.

Proof of Theorem 5.1. (1) It is easy to verify from the energy (1.2) and Eq. (1.1) that

$$
\frac{d}{d t} E(h(t))=-f_{\Omega} h_{t}^{2} d x \leq 0 \quad \forall t>0 .
$$

Thus, the energy decays. Consequently, we have by (5.2) that

$$
E(h(t)) \leq E\left(h\left(t_{\xi}\right)\right)=-\log (\xi L) \quad \forall t \geq t_{\xi} .
$$

This and Jensen's inequality imply that

$$
\begin{aligned}
-\log (\xi L) & =f_{t_{\xi}}^{t} E\left(h\left(t_{\xi}\right)\right) d \tau \\
& \geq f_{t_{\xi}}^{t} E(h(\tau)) d \tau \\
& \geq f_{t_{\xi}}^{t} f_{\Omega}\left[-\frac{1}{2} \log \left(1+|\nabla h(x, \tau)|^{2}\right)\right] d x d \tau \\
& \geq-\frac{1}{2} \log \left[1+f_{t_{\xi}}^{t} f_{\Omega}|\nabla h(x, \tau)|^{2} d x d \tau\right] .
\end{aligned}
$$

Therefore, applying an integration by parts and the Cauchy-Schwarz inequality, we obtain by (2.3) that

$$
\begin{aligned}
(\xi L)^{2} & \leq 1+f_{t_{\xi}}^{t} f_{\Omega}|\nabla h(x, \tau)|^{2} d x d \tau \\
& =1+f_{t_{\xi}}^{t} f_{\Omega}[-h(x, \tau)] \Delta h(x, \tau) d x d \tau
\end{aligned}
$$




$$
\begin{aligned}
& \leq 1+\left(f_{t_{\xi}}^{t} f_{\Omega}|h(x, \tau)|^{2} d x d \tau\right)^{1 / 2}\left(f_{t_{\xi}}^{t} f_{\Omega}|\Delta h(x, \tau)|^{2} d x d \tau\right)^{1 / 2} \\
& \leq 1+\left(f_{t_{\xi}}^{t}\left[w_{h}(\tau)\right]^{2} d \tau\right)^{1 / 2}\left(1+\frac{\left[w_{h}\left(t_{\xi}\right)\right]^{2}}{2\left(t-t_{\xi}\right)}\right)^{1 / 2} .
\end{aligned}
$$

Now, if $t \geq 2 t_{\xi}+(1 / 2)\left[w_{h}(0)\right]^{2}$, then by $(2.2)$

$$
1+\frac{\left[w_{h}\left(t_{\xi}\right)\right]^{2}}{2\left(t-t_{\xi}\right)} \leq 1+\frac{2 t_{\xi}+\left[w_{h}(0)\right]^{2}}{2\left(t_{\xi}+\frac{1}{2}\left[w_{h}(0)\right]^{2}\right.}=2 .
$$

Combining (5.7), (5.8), and the assumption that $\xi L \geq \sqrt{2}$, we obtain (5.3).

(2) Setting $t_{0}=0$ in (2.5), by (5.6) and (5.2), we have for any $t>\left[w_{h}(0)\right]^{2}$ that

$$
\begin{aligned}
-\frac{1}{2} \log (1+\sqrt{3 t}) & \leq \int_{0}^{t} E(h(\tau)) d \tau \\
& =\frac{1}{t} \int_{0}^{t_{\xi}} E(h(\tau)) d \tau+\frac{1}{t} \int_{t_{\xi}}^{t} E(h(\tau)) d \tau \\
& \leq \frac{t_{\xi}}{t} E(h(0))-\frac{t-t_{\xi}}{t} \log (\xi L) .
\end{aligned}
$$

Consequently, if $t=\sigma t_{\xi}$ with $\sigma>1$, then

$$
-\frac{1}{2} \log (1+\sqrt{3 t}) \leq \frac{1}{\sigma} E(h(0))-\frac{\sigma-1}{\sigma} \log (\xi L) .
$$

Thus, for $t \geq 1 / 3$,

$$
2 \sqrt{3 t} \geq 1+\sqrt{3 t} \geq e^{-\frac{2}{\sigma} E(h(0))}(\xi L)^{\frac{2(\sigma-1)}{\sigma}} .
$$

This leads to (5.4). Q.E.D.

Acknowledgment. This work was partially supported by the NSF through grant DMS0072958 and DMS-0107218. The authors thank Dr. Robert L. Pego for many interesting discussions. They also thank the referee for the insightful and suggestive comments.

\section{References}

[1] R. Adams. Sobolev Spaces. Academic Press, New York, 1975.

[2] J. G. Amar and F. Family. Critical temperature for mound formation in molecularbeam-epitaxy. Phys. Rev. B, 54(19):14071-14076, 1996.

[3] J. G. Amar and F. Family. Effects of crystalline microstructure on epitaxial growth. Phys. Rev. B, 54(20):14742-14753, 1996. 
[4] G. S. Bales and A. Zangwill. Morphological instability of a terrace edge during step-flow growth. Phys. Rev. B, 41(9):5500-5508, 1990.

[5] G. Ehrlich and F. G. Hudda. Atomic view of surface diffusion: Tungsten on tungsten. J. Chem. Phys., 44:1036, 1966.

[6] H.-J. Ernst, F. Fabre, R. Folkerts, and J. Lapujoulade. Observation of a growth instability during low temperature molecular beam epitaxy. Phys. Rev. Lett., 72(1):112-115, 1994.

[7] L. C. Evans. Partial Differential Equations, volume 19 of Graduate Studies in Mathematics. AMS, Providence, 1998.

[8] L. Golubović. Interfacial coarsening in epitaxial growth models without slope selection. Phys. Rev. Lett., 78(1):90-93, 1997.

[9] M. F. Gyure, J. J. Zinck, C. Ratsch, and D. D. Vvedensky. Unstable growth on rough surfaces. Phys. Rev. Lett., 81(22):4931-4934, 1998.

[10] C. Herring. Surface tension as a motivation for sintering. In W. E. Kingston, editor, The Physics of Powder Metallurgy, pages 143-179. McGraw-Hill, 1951.

[11] A. W. Hunt, C. Orme, D. R. M. Williams, B. G. Orr, and L. M. Sander. Instabilities in MBE growth. Europhys. Lett., 27:611, 1994.

[12] M. D. Johnson, C. Orme, A. W. Hunt, D. Graff, J. Sudijono, L. M. Sander, and B. G. Orr. Stable and unstable growth in molecular beam epitaxy. Phys. Rev. Lett., 72(1):116119, 1994.

[13] M. Kardar, G. Parisi, and Y. C. Zhang. Dynamic scaling of growing interfaces. Phys. Rev. Lett., 56:889-892, 1986.

[14] R. V. Kohn and F. Otto. Upper bounds on coarsening rates. Commun. Math. Phys., 229:375-395, 2002.

[15] R. V. Kohn and X. Yan. Upper bounds on the coarsening rate for an epitaxial growth model. Commun. Pure Appl. Math., 56(11):1549-1564, 2003.

[16] J. Krug. Origins of scale invariance in growth processes. Adv. Phys., 46:139-282, 1997.

[17] J. Krug, M. Plischke, and M. Siegert. Surface diffusion currents and the universality classes of growth. Phys. Rev. Lett., 70(21):3271-3274, 1993.

[18] J. Krug and M. Schimschak. Metastability of step flow growth in $1+1$ dimensions. $J$. Phys. I France, 5:1065-1086, 1995. 
[19] Z.-W. Lai and S. Das Sarma. Kinetic growth with surface relaxation: Continuum versus atomistic models. Phys. Rev. Lett., 66:2348-2351, 1991.

[20] B. Li and J.-G. Liu. Thin film epitaxy with or without slope selection. European J. Applied Math., 14(6):713-743, 2003.

[21] D. Moldovan and L. Golubović. Interfacial coarsening dynamics in epitaxial growth with slope selection. Phys. Rev. E, 61(6):6190-6214, 2000.

[22] W. W. Mullins. Theory of thermal grooving. J. Appl. Phys., 28:333-339, 1957.

[23] M. Ortiz, E. Repetto, and H. Si. A continuum model of kinetic roughening and coarsening in thin films. J. Mech. Phys. Solids, 47:697-730, 1999.

[24] P. Politi and A. Torcini. Coarsening in surface growth models without slope selection. J. Phys. A: Math. Gen., 33:L77-L82, 2000.

[25] P. Politi and J. Villain. Ehrlich-Schwoebel instability in molecular-beam epitaxy: A minimal model. Phys. Rev. B, 54(7):5114-5129, 1996.

[26] I. Raistrick and M. Hawley. Scanning tunneling and atomic force microscope studies of thin sputtered films of $\mathrm{YBa}_{2} \mathrm{Cu}_{3} \mathrm{O}_{7}$. In S. L. Shinde and D. A. Rudman, editors, Interfaces in High-Tc Superconducting Systems, pages 28-70, New York, 1994. SpringerVerlag.

[27] A. Roshko, F. Stork, D. Rudman, D. Aldrich, and P. M. Hotsenpiller. Comparison of heteroepitaxial $\mathrm{YBa}_{2} \mathrm{Cu}_{3} \mathrm{O}_{7-\delta}$ and $\mathrm{TiO}$ thin film growth. J. Crystal Growth, 174:398408, 1997.

[28] M. Rost and J. Krug. Coasening of surface structures in unstable epitaxial growth. Phys. Rev. E, 55(4):3952-3957, 1997.

[29] M. Rost, P. Šmilauer, and J. Krug. Unstable epitaxy on vicinal surfaces. Surface Sci., 369:393-402, 1996.

[30] R. L. Schwoebel. Step motion on crystal surfaces II. J. Appl. Phys., 40:614, 1969.

[31] R. L. Schwoebel and E. J. Shipsey. Step motion on crystal surfaces. J. Appl. Phys., 37:3682, 1966 .

[32] M. Siegert. Coarsening dynamics in crystalline thin films. Phys. Rev. Lett., 81(25):54815484, 1998.

[33] M. Siegert and M. Plischke. Slope selection and coarsening in molecular beam epitaxy. Phys. Rev. Lett., 73(11):1517-1520, 1994. 
[34] J. A. Stroscio, D. T. Pierce, M. D. Stiles, and A. Zangwill. Coarsening of unstable surface features during Fe(001) homoepitaxy. Phys. Rev. Lett., 75(23):4246-4249, 1995.

[35] K. Thürmer, R. Koch, M. Weber, and K. H. Rieder. Dynamic evolution of pyramid structures during growth of epitaxial Fe(001) films. Phys. Rev. Lett., 75(9):1767-1770, 1995.

[36] J. Villain. Continuum models of crystal growth from atomic beams with and without desorption. J. de Phys. I, 1:19-42, 1991.

[37] D. D. Vvedensky, A. Zangwill, C. N. Luse, and M. R. Wilby. Stochastic equations of motion for epitaxial growth. Phys. Rev. E, 48:852-862, 1993. 